\title{
Prevalencia de Bruxismo y Trastornos Temporomandibulares Asociados en una Población de Escolares de San Luis Potosí, México
}

\author{
Prevalence of Bruxism and Associated Temporomandibular Disorders in a Population of \\ School Children of San Luis Potosí, México
}

Emilio R. Rodríguez Robledo; Ricardo Martínez Rider; Ma. Del Socorro Ruiz Rodríguez; Raúl Márquez Preciado; J. Arturo Garrocho Rangel; Amaury de Jesús Pozos Guillén \& Miguel Ángel Rosales Berber

RODRÍGUEZ, R. E. R.; MARTíNEZ, R. R.; RUIZ, R. M. S.; MÁRQUEZ, P. R.; GARROCHO, R. J. A.; POZOS, G. A. J. \& ROSALES, B. M. Á. Prevalencia de bruxismo y trastornos temporomandibulares asociados en una población de escolares de San Luis Potosí, México. Int. J. Odontostomat., 12(4):382-387, 2018.

RESUMEN: El término "Transtornos Temporomandibulares" (TTM) incluye numerosos problemas clínicos asociados con las articulaciones temporomandibulares, músculos de la masticación y otras estructuras asociadas. El bruxismo, un hábito oral parafuncional, consiste en rechinar o apretar de manera rítmica e involuntaria los dientes, lo que puede conducir a trauma oclusal y problemas articulares. El objetivo del estudio fue determinar la frecuencia y prevalencia de signos y síntomas de TTM y bruxismo en niños escolares de 6 a 12 años, en una población de San Luis Potosí, México. Se empleó un diseño transversal descriptivo en una muestra de 314 participantes, estudiantes de una escuela primaria de la ciudad de San Luis Potosí, México. Los participantes fueron seleccionados aleatoriamente en forma estratificada y polietápica. La muestra total consistió en 153 femeninos y 161 masculinos. Los signos y síntomas de TTM más frecuentes fueron la alteración de la función de la ATM (35\%), seguido por los ruidos y chasquido articulares $(29,2 \%)$. En los sujetos con diagnóstico de bruxismo, la alteración más frecuente fueron molestias asociadas en la ATM (19,4 \%), dolor de cabeza (17,8 \%) y atrición dental (16,5\%).

PALABRAS CLAVE: transtornos temporomandibulares, bruxismo, prevalencia, chasquido articular.

\section{INTRODUCCIÓN}

La articulación temporomandibular (ATM) es una articulación ginglimoartrodial bilateral constituida por el hueso temporal, el disco articular y la mandíbula que permite movimientos funcionales de la boca, como apertura, cierre, masticación, fonación, entre otros (Moreno Silva, 2013). La Academia Americana de Odontología Pediátrica (AAPD) define a los Trastornos de la Articulación Temporomandibular (TTM) como un grupo de alteraciones musculoesqueléticas y neuromusculares que incluye diversos signos y síntomas clínicos, que involucran a la ATM y sus estructuras asociadas (American Academy of Pediatric Dentistry, 2015).

Los TTM afectan a más del $50 \%$ de la población mundial, sin predilección por sexo; aunque son más frecuentes en los adultos, se pueden manifestar a cual- quier edad (Serrano et al., 2009); a lo largo de los últimos años, la prevalencia de TTM ha ido en aumento y es cada vez más común entre los niños y adolescentes (Sena et al., 2013).

Por otra parte, el bruxismo es un grupo de hábitos parafuncionales orales asociado comúnmente con movimientos rítmicos e inconscientes de la mandíbula y rechinamiento dental, que se considera factor de riesgo para los TTM (de la Hoz-Aizpurua et al., 2011). El bruxismo se ha clasificado como nocturno (bruxismo del sueño) que se realiza inconscientemente durante el sueño, y que ha sido asociada con sueño intranquilo, anomalías musculares y neurológicas; y el bruxismo diurno, cuya manifestación preponderante es el apretamiento entre ambas arcadas dentarias; es una 
hiperactividad inducida por generalmente por aspectos psicosociales, como el estrés y la ansiedad (Seraj et al., 2009; Bayar et al., 2012; Wieckiewicz et al., 2014). El bruxismo del sueño se ha asociado con el incremento de la actividad neuromuscular mandibular y de otras áreas corporales, del ritmo cardiaco, y con el aumento de la frecuencia y profundidad respiratorias (Sandoval \& Fariña, 2016). Otros factores de tipo morfológico que intervienen en esta anomalía son las discrepancias oclusales y las interrelaciones anatómicas de las estructuras óseas de la región orofacial (Castellanos, 2015).

El bruxismo tiene una prevalencia en la población adulta general reportada entre el $10 \%$ al $20 \%$, y en niños varía entre el $7 \%$ y el $15,1 \%$ (Seraj et al.). Se puede sospechar de la presencia de bruxismo cuando hay evidencia clara de facetas de desgaste en las caras oclusales que no pueden ser interpretadas como resultado de la función masticatoria normal (Lavigne et al., 2003; Bayar et al.).

Durante mucho tiempo se consideró que los TTM eran una situación ajena al niño; sin embargo, múltiples investigaciones han reportado hallazgos que sugieren que este trastorno es más frecuente en la población pediátrica de lo que se pensaba. Por esta razón, se ha tratado de identificar cuáles son las condiciones anormales de la ATM más comunes en el niño, con el fin de conocer el posible efecto de las maloclusiones dentarias a edades tempranas y su progreso hacia la adultez (Arenas Carreño et al., 2013). Esto significa que los TTM pueden prevenirse con un tratamiento oportuno basado en un diagnóstico integral. Sin embargo, se ha prestado poca atención a la presencia de tales patologías funcionales en los niños y adolescentes jóvenes, debido en parte a la dificultad del proceso de valoración funcional articular y al gran potencial de adaptabilidad que exhiben las estructuras articulares, lo cual podría enmascarar ciertas TTM (Campos et al., 2006). En este contexto, el propósito del presente estudio transversal fue calcular la prevalencia de bruxismo y determinar cuáles son los principales signos y síntomas de TTM asociados a esta condición en una muestra de niños escolares representativa de la ciudad de San Luis Potosí, México.

\section{MATERIAL Y MÉTODO}

El estudio fue de tipo transversal descriptivo, que se realizó en una muestra de 314 alumnos de enseñanza básica de una escuela primaria de la ciudad de
San Luis Potosí, México. El protocolo fue aprobado por un Comité de Ética de Investigación (Facultad de Estomatología. Universidad Autónoma de San Luis Potosí, México).

La selección de los participantes se llevó a cabo de manera estratificada por género y en forma polietápica. De los 18 grupos escolares totales, se seleccionaron aleatoriamente 9; posteriormente, de cada uno de estos 8 grupos se seleccionaron 35 estudiantes, para una muestra total de 315 participantes. Sin embargo, los padres de 1 de estos estudiantes no aceptaron participar. Los criterios de inclusión fueron: cualquier género, edad entre 6 y 12 años, sanos sistémicamente y la aceptación para participar mediante la firma de un consentimiento informado por parte de los padres o representantes legales de cada niño.

Todos los alumnos seleccionados fueron revisados y evaluados por un único investigador, previamente estandarizado para los procedimientos diagnósticos de interés. Los datos fueron registrados y clasificados por edad y sexo, en dos instrumentos de recolección de datos, en relación a las variables "signos y síntomas de TTM" y "bruxismo". Para TTM se obtuvo información acerca de (1) alteración de la función de la ATM (sin dolor, pero clínicamente se observa un "brinco" durante la función), (2) ruidos o chasquidos, (3) limitación de la apertura bucal, (4) desviaciones durante la apertura o cierre, (5) limitación de los movimientos de lateralidad, (6) limitación de los movimientos de protrusión, y (7) dolor en la ATM. En cuanto al diagnóstico de bruxismo, la siguiente información fue recabada: (1) dolor de cabeza, (2) dolor crónico en la ATM, (3) dolor muscular durante la masticación, (4) tono muscular alterado, y (5) atrición dental evidente. Dentro de cada categoría se registraron frecuencias y porcentajes, divididos en tres grupos de edad: 6 a 7 años 11 meses, 8 a 9 años 11 meses, 10 a 12 años.

\section{RESULTADOS}

La muestra de participantes en el presente estudio estuvo conformada por 314 estudiantes de 6 a 12 años, de los cuales 161 fueron masculinos (51\%) y 153 femeninos (49\%). El signo de TTM más frecuente fue la alteración de la función de la ATM en 109 (34 \%) alumnos, principalmente del sexo masculino, seguido por los ruidos y chasquidos articulares en 102 participantes $(32,4 \%)$, en los que el sexo femenino fue el más afectado (Tabla I). 
Tabla I. Signos y síntomas de TTM del total de la muestra

\begin{tabular}{lcccccccc}
\hline $\begin{array}{l}\text { Total de la } \\
\text { muestra }\end{array}$ & $\begin{array}{c}\text { Alteración } \\
\text { de la } \\
\text { función }\end{array}$ & Chasquido & $\begin{array}{c}\text { Limitación } \\
\text { apertura }\end{array}$ & $\begin{array}{c}\text { Desviación } \\
\text { apertura /cierre }\end{array}$ & $\begin{array}{c}\text { Limitación } \\
\text { lateralidad }\end{array}$ & $\begin{array}{c}\text { Limitación } \\
\text { protrusión }\end{array}$ & $\begin{array}{c}\text { Dolor } \\
\text { ATM }\end{array}$ & $\begin{array}{c}\text { Total } \\
\text { (a) }\end{array}$ \\
\hline Masculino & 58 & 48 & 15 & 48 & 27 & 35 & 6 & 237 \\
Femenino 153 & 58 & 54 & 15 & 36 & 21 & 27 & 10 & 214 \\
Total 314 & 109 & 102 & 30 & 84 & 48 & 62 & 16 & 451 \\
\hline
\end{tabular}

Entre los signos y síntomas del bruxismo la alteración más frecuentes fue el dolor de origen articular en 61 alumnos (19,4\%); el sexo más afectado fue el masculino. También se registraron dolores de cabeza en 56 sujetos (17,8\%) y atrición dentaria en 52 individuos $(16,5 \%)$ (Tabla II).

Los signos y síntomas de DTM y de Bruxismo por grupo de edad se describen en las siguientes tablas:
Signos y síntomas de TTM de 6 a 7 años 11 meses (Tabla III).

Signos y síntomas de Bruxismo de 6 a 7 años 11 meses (Tabla IV).

Signos y síntomas de TTM de 8 a 9 años 11 meses (Tabla V).

Signos y síntomas de Bruxismo de 8 a 9 años 11 meses (Tabla VI).

Signos y síntomas de TTM de 10 a 12 años (Tabla VII). Signos y síntomas de Bruxismo de10 a12 años (Tabla VIII).

Tabla II. Signos y síntomas de Bruxismo del total de la muestra.

\begin{tabular}{lcccccc}
\hline Total de la muestra & $\begin{array}{c}\text { Dolor de } \\
\text { cabeza }\end{array}$ & $\begin{array}{c}\text { Dolor en } \\
\text { ATM }\end{array}$ & $\begin{array}{c}\text { Dolor músculos } \\
\text { masticación }\end{array}$ & $\begin{array}{c}\text { Tono muscular } \\
\text { alterado }\end{array}$ & Atrición & Total \\
\hline Masculino 161 & 28 & 36 & 23 & 14 & 31 & 132 \\
Femenino 153 & 28 & 25 & 12 & 4 & 21 & 90 \\
Total 314 & 56 & 61 & 35 & 18 & 52 & 222 \\
\hline
\end{tabular}

Tabla III. Signos y síntomas de TTM en niños de 6 a 7 años 11 meses.

\begin{tabular}{|c|c|c|c|c|c|c|c|c|}
\hline $\begin{array}{c}6 \text { a } 7 \text { años } 11 \\
\text { meses }\end{array}$ & $\begin{array}{l}\text { Alteración de } \\
\text { la función }\end{array}$ & Chasquido & $\begin{array}{c}\text { Limitación } \\
\text { apertura }\end{array}$ & $\begin{array}{c}\text { Desviación } \\
\text { apertura /cierre }\end{array}$ & $\begin{array}{l}\text { Limitación } \\
\text { lateralidad }\end{array}$ & $\begin{array}{l}\text { Limitación } \\
\text { protrusión }\end{array}$ & $\begin{array}{l}\text { Dolor } \\
\text { ATM }\end{array}$ & Total \\
\hline Masculino 51 & 15 & 20 & 4 & 6 & 6 & 6 & 3 & 60 \\
\hline Femenino 59 & 11 & 13 & 2 & 4 & 5 & 5 & 0 & 40 \\
\hline Total 110 & 26 & 33 & 6 & 10 & 11 & 11 & 3 & 100 \\
\hline
\end{tabular}

Tabla IV. Signos y síntomas de bruxismo en niños de 6 a 7 años 11 meses.

\begin{tabular}{lcccccc}
\hline 6 a 7años 11 meses & $\begin{array}{c}\text { Dolor de } \\
\text { cabeza }\end{array}$ & Dolor en ATM & $\begin{array}{c}\text { Dolor músculos } \\
\text { masticación }\end{array}$ & $\begin{array}{c}\text { Tono muscular } \\
\text { alterado }\end{array}$ & Atrición & Total \\
\hline Masculino 51 & 2 & 5 & 5 & 2 & 7 & 21 \\
Femenino 59 & 5 & 3 & 5 & 1 & 7 & 21 \\
Total 110 & 7 & 8 & 10 & 3 & 14 & 42
\end{tabular}

Tabla V. Signos y síntomas de TTM en niños de 8 a 9 años 11 meses.

\begin{tabular}{|c|c|c|c|c|c|c|c|c|}
\hline $\begin{array}{l}8 \text { a } 9 \text { años } \\
11 \text { meses }\end{array}$ & $\begin{array}{l}\text { Alteración de } \\
\text { la función }\end{array}$ & Chasquido & $\begin{array}{l}\text { Limitación } \\
\text { apertura }\end{array}$ & $\begin{array}{c}\text { Desviación } \\
\text { apertura / cierre }\end{array}$ & $\begin{array}{l}\text { Limitación } \\
\text { lateralidad }\end{array}$ & $\begin{array}{l}\text { Limitación } \\
\text { prot rusión }\end{array}$ & $\begin{array}{l}\text { Dolor } \\
\text { ATM }\end{array}$ & Total \\
\hline Masculino & 22 & 19 & 9 & 24 & 15 & 18 & 1 & 108 \\
\hline Femenino 39 & 23 & 13 & 3 & 18 & 11 & 10 & 0 & 78 \\
\hline Total 96 & 45 & 32 & 12 & 42 & 26 & 28 & 1 & 186 \\
\hline
\end{tabular}

Tabla VI. Signos y síntomas de bruxismo en niños de 8 a 9 años 11 meses.

\begin{tabular}{lcccccc}
\hline 8 a 9 años 11 meses & $\begin{array}{c}\text { Dolor de } \\
\text { cabeza }\end{array}$ & $\begin{array}{c}\text { Dolor en } \\
\text { ATM }\end{array}$ & $\begin{array}{c}\text { Dolor músculos } \\
\text { masticación }\end{array}$ & $\begin{array}{c}\text { Tono muscular } \\
\text { alterado }\end{array}$ & Atrición & Total \\
\hline Masculino 57 & 13 & 17 & 12 & 10 & 11 & 63 \\
Femenino 39 & 16 & 16 & 3 & 3 & 6 & 44 \\
Total 96 & 29 & 33 & 15 & 13 & 17 & 107 \\
\hline
\end{tabular}


RODRÍGUEZ, R. E. R.; MARTíNEZ, R. R.; RUIZ, R. M. S.; MÁRQUEZ, P. R.; GARROCHO, R. J. A.; POZOS, G. A. J. \& ROSALES, B. M. Á. Prevalencia de bruxismo y trastornos temporomandibulares asociados en una población de escolares de San Luis Potosí, México. Int. J. Odontostomat., 12(4):382-387, 2018.

Tabla VII. Signos y síntomas de TTM en niños de 10 a 12 años.

\begin{tabular}{lcccccccc}
\hline 10 a 12 años & $\begin{array}{c}\text { Alteración } \\
\text { funciónal }\end{array}$ & Chasquido & $\begin{array}{c}\text { Limitación } \\
\text { apertura }\end{array}$ & $\begin{array}{c}\text { Desviación } \\
\text { apertura /cierre }\end{array}$ & $\begin{array}{c}\text { Limitación } \\
\text { lateralidad }\end{array}$ & $\begin{array}{c}\text { Limitación } \\
\text { protrusión }\end{array}$ & $\begin{array}{c}\text { Dolor } \\
\text { ATM }\end{array}$ & $\begin{array}{c}\text { Total } \\
\text { Masculino } 21\end{array}$ \\
\hline 21 & 9 & 6 & 18 & 6 & 11 & 2 & 73 \\
Femenino 17 & 17 & 18 & 10 & 14 & 5 & 12 & 10 & 86 \\
Total 123 & 38 & 27 & 16 & 34 & 11 & 23 & 12 & 159 \\
\hline
\end{tabular}

Tabla VIII. Signos y síntomas de bruxismo en niños de 10 a 12 años.

\begin{tabular}{lcccccc}
\hline 10 a 12 años & $\begin{array}{c}\text { Dolor de } \\
\text { cabeza }\end{array}$ & $\begin{array}{c}\text { Dolor en } \\
\text { ATM }\end{array}$ & $\begin{array}{c}\text { Dolor músculos } \\
\text { masticación }\end{array}$ & $\begin{array}{c}\text { Tono muscular } \\
\text { alterado }\end{array}$ & Atrición & Total \\
\hline Masculino 60 & 13 & 14 & 6 & 2 & 13 & 46 \\
Femenino 63 & 7 & 6 & 4 & 0 & 8 & 24 \\
Total 123 & 18 & 20 & 10 & 2 & 20 & 70 \\
\hline
\end{tabular}

\section{DISCUSIÓN}

Los TTM son una condición multifactorial que abarca a una miríada de problemas clínicos específicos, que involucran principalmente la articulación temporomandibular, la musculatura masticatoria o ambos, con la probable participación de otros factores contribuyentes (McNeill et al., 1997; Sena et al.). El objetivo del presente estudio fue evaluar la frecuencia de los signos y síntomas de TTM y bruxismo en una muestra de niños escolares, de 6 a 12 años en la ciudad de San Luis Potosí, México. Nuestros resultados indican que los signos y síntomas de TTM más frecuentemente fueron la alteración de la función (34,7 $\%$ ) y el chasquido articular (32,4 \%). Estos hallazgos son concordantes con los reportados por Arenas Carreño et al., Barone et al. (1997) y Tuerlings \& Limme (2004), donde mencionan que el chasquido articular y el dolor de cabeza son los síntomas más comunes en los casos de TTM en niños y adolescentes, seguido por el patrón alterado de apertura/cierre mandibular (Alamoudi, 2001; Arenas Carreño et al.). Así mismo, en su estudio con 300 pacientes pediátricos, menciona que el síntoma más común entre los afectados por TTM fue el chasquido articular. En contraste, Faribay \& Mohammadi (2005) reportaron una prevalencia de TTM de $11.7 \%$ en una muestra similar de estudiantes iraníes, con rango de edad similar al mencionado aquí.

En general, la literatura dental ha establecido que la prevalencia de los TTM presenta un rango muy amplio de valores en pacientes pediátricos, desde un $16 \%$ en edades de dentición primaria hasta un $90 \%$ durante la etapa de dentición mixta (Castelo et al., 2005; Riva et al., 2010). En este mismo contexto, algunos estudios han demostrado que la frecuencia de signos y síntomas de TTM tiende a incrementarse con la edad, lo cual puede ser explicado por el hecho de a mayor edad las tensiones musculares son más inten- sas y de mayor duración. Tales tensiones producen cambios intracapsulares, que afectan el funcionamiento de la articulación temporomandibular (Karibe et al., 2015; Friedman Rubin et al., 2018). Sin embargo, otros estudios, al igual que el nuestro, no han confirmado esta relación incremento de signos/síntomas de TTM con la edad del paciente (Anastassaki Köhler et al., 2012).

De acuerdo a Barbosa et al. (2008), uno de los aspectos más controversiales cuando se estudian los TTM en niños y adolescentes es la determinación de los factores causales, puesto que esta condición es altamente heterogénea entre los pacientes. Al respecto, deben siempre considerarse durante la entrevista inicial la posible influencia de perturbaciones emocionales o psicosociales y también de diferentes hábitos parafuncionales, especialmente el bruxismo (Widmalm et al., 1995; List et al., 2001; Karibe et al.). Este hábito se define como la presencia sostenida, repetitiva e involuntaria de presiones indeseables de la mandíbula sobre el sistema masticatorio (Rugh \& Harlan, 1988; Riva et al., 2010; Castellanos et al.; Sandoval \& Fariña, 2016). Como se mencionó anteriormente, existen dos tipos de bruxismo: el diurno y el del sueño. El primero exhibe una prevalencia general del $20 \%$ y está fuertemente relacionado al estrés o ansiedad, mientras que el segundo tipo, cuya prevalencia se ha reportado en un $8 \%$, puede aparecer a edades tan tempranas como el primer año de vida, con la erupción de los incisivos inferiores permanentes (Souza et al., 2008). Sin embargo, la asociación entre la ocurrencia de estos hábitos parafuncionales y la presencia de TTM es aún controversial, por lo que no se ha confirmado plenamente hasta la actualidad (de la Hoz-Aizpurua et al.; Sandoval \& Fariña; Friedman Rubin et al.). Por otra parte, se ha mencionado que el género femenino tiende a exhibir una mayor prevalencia de signos y síntomas de TTM (Winocur et al., 2006). Este hallazgo puede ser explicado por el hecho de que las mujeres tienden a mostrar un menor umbral al dolor, ade- 
más de otros factores, como los cambios hormonales tales como la progesterona (Friedman Rubin et al.). Sin embargo, en el presente estudio no se pudo observar una diferencia entre prevalencia de acuerdo al género de los participantes; resultados similares fueron reportados por Emodi-Perlman et al. (2012) y Friedman Rubin et al.

Al igual que otros estudios con muestras similares de participantes, el presente trabajo no estuvo exento de limitaciones. Las más importantes que se pueden mencionar son: (1) La muestra estudiada solamente se obtuvo de una única escuela y no se seleccionó de forma aleatoria, sino por conveniencia; (2) Las examinaciones realizadas en los escolares fue realizada en la misma escuela, no en una clínica dental bajo condiciones ideales; (3) No se realizó una valoración psicológica de los participantes; y (4) Durante la inspección clínica de los participantes no se contó con la presencia de los padres, los cuales pudieron aportar mayor información útil asociada a los propósitos del estudio.

Como conclusión, en nuestra muestra estudiada de niños y adolescentes escolares los signos y síntomas de TTM más frecuentemente encontrados fueron la alteración de la función de la ATM, seguido por los ruidos y chasquido articulares. En aquellos sujetos diagnosticados con bruxismo, las alteraciones más frecuentes fueron sensibilidad de la ATM, dolor de cabeza y atrición dental.

Ante la alta frecuencia de signos y síntomas de DTM y bruxismo reportada aquí, al igual que en otros estudios previos similares, se debe enfatizar que una de las obligaciones del odontólogo pediatra es implementar estrategias para prevenir e interceptar las anomalías funcionales de la articulación temporomandibular, ya desde edades tempranas. Sin embargo, aún no existe un consenso general acerca de los criterios diagnósticos para la detección de los problemas articulares en los pacientes pediátricos. Aun así, no debemos dejar pasar por alto cualquier signo o síntoma que pueda ser indicativo de la presencia de TTM, como son dolor localizado en el área de la ATM, cefalea, otalgia o dolor de los músculos masticatorios, apertura bucal limitada, desgastes dentarios anormales, desviaciones mandibulares a la apertura/cierre, presencia de ruidos articulares, entre otros.

Por lo tanto, se recomienda realizar un examen minucioso del área de la articulación temporomandibular, para establecer con precisión un buen diagnóstico de TTM que ayude a realizar un tratamiento exitoso. El manejo correspondiente de las TTM en pacientes pediátricos es multidisciplinario, en el que deben participar psicólogos, psiquiatras, especialistas en alteraciones del sueño, neurólogos, internistas, rehabilitadores orales, cirujanos maxilofaciales y, por supuesto, el odontólogo pediatra.

RODRÍGUEZ, R. E. R.; MARTÍNEZ, R. R.; RUIZ, R. M. S.; MÁRQUEZ, P. R.; GARROCHO, R. J. A.; POZOS, G. A. J. \& ROSALES, B. M. Á. Prevalence of bruxism and temporomandibular associated disorders in a population of school children of San Luis Potosí, México. Int. J. Odontostomat., 12(4):382-387, 2018.

ABSTRACT: The term "Temporomandibular disorders" (TTM) includes numerous clinical problems associated with joint temporomandibular, muscles of mastication and other associated structures. Bruxism, oral parafunctional habit, consists of grinding or clenching of involuntary, rhythmic manner, which can lead to trauma, occlusal and problems joint. The objective of the study was to determine the frequency and prevalence of signs and symptoms of DTM and bruxism in school children aged 6 to 12 , in a population of San Luis Potosí, México. A crosssectional descriptive design was used in a sample of 314 participants, students of a primary school in the city of San Luis Potosi, Mexico. The participants were randomly selected in tiered form and conglomerated. The total sample consisted of 153 female and male 161 . The signs and symptoms of TMD frequent were alteration of the function of the ATM (35 $\%$ ), followed by noise and snap joint $(29.2 \%)$. In subjects with a diagnosis of bruxism, the most frequent alteration was associated with TMJ discomfort (19.4\%), headache (17.8 $\%)$ and dental attrition (16.5\%).

KEY WORDS: temporomandibular disorders, bruxism, prevalence, articular clicking.

\section{REFERENCIAS BIBLIOGRÁFICAS}

Alamoudi, N. Correlation between oral parafunction and temporomandibular disorders and emotional status among saudi children. J. Clin. Pediatr. Dent., 26(1):71-80, 2001.

American Academy of Pediatric Dentistry. Guideline on Acquired Temporomandibular Disorders in Infants, Children, and Adolescents. En: American Academy of Pediatric Dentistry. Clinical Practice Guidelines, Reference Manual, 37(6):272-8, 2015. Disponible en: http://www.aapd.org/media/ Policies_Guidelines/G_TMD.pdf

Anastassaki Köhler, A.; Hugoson, A. \& Magnusson, T. Prevalence of symptoms indicative of temporomandibular disorders in adults: cross-sectional epidemiological investigations covering two decades. Acta Odontol. Scand., 70(3):213-23, 2012.

Arenas Carreño, M. A.; Bloise Triana, A.; Carvajal Pabón, M. E.; Forero Santamaría, C. E.; Rodríguez Ciódaro, A. \& Herrera Vi- 
vas, M. C. Signos y síntomas de trastornos temporomandibulares en niños entre los 6 y los 13 años de edad. Serie de 50 casos. Univ Odontol., 32(69):161-8, 2013.

Barbosa, T. de S.; Miyakoda, L.; Pocztaruk, R. de L.; Rocha, C. P. \& Gavião, M. B. Temporomandibular disorders and bruxism in childhood and adolescence: review of the literature. Int. J. Pediatr. Otorhinolaryngol., 72(3):299-314, 2008.

Barone, A.; Sbordone, L. \& Ramaglia, L. Craniomandibular disorders and orthodontic treatment need in children. J. Oral Rehabil., 24(1):2-7, 1997.

Bayar, G. R.; Tutuncu, R. \& Acikel, C. Psychopathological profile of patients with different forms of bruxism. Clin. Oral Investig., 16(1):305-11, 2012.

Campos, M.; Herrera A. \& Ruan, V. Desórdenes temporomandibulares en la población infantil. Un tema controversial - Revisión bibliográfica. Rev. Latinoam. Ortod. Odontopediatr., 2006. Disponible en: https://www.ortodoncia.ws/publicaciones/2006/art-8/

Castellanos, J. L. Bruxismo. Nociones y conceptos. Rev. A. D. M., 72(2):63-9, 2015.

Castelo, P. M.; Gavião, M. B.; Pereira, L. J. \& Bonjardim L. R. Relationship between oral parafunctional/nutritive sucking habits and temporomandibular joint dysfunction in primary dentition. Int. J. Paediatr. Dent., 15(1):29-36, 2005.

de la Hoz-Aizpurua, J. L.; Díaz-Alonso, E.; LaTouche-Arbizu, R. \& Mesa-Jiménez, J. Sleep bruxism. Conceptual review and update. Med. Oral Patol. Oral Cir. Bucal, 16(2):e231-8, 2011.

Emodi-Perlman, A.; Eli, I.; Friedman-Rubin, P.; Goldsmith, C.; Reiter, S. \& Winocur, E. Bruxism, oral parafunctions, anamnestic and clinical findings of temporomandibular disorders in children. J. Oral Rehabil., 39(2):126-35, 2012.

Faribay, J. \& Mohammadi, M. Prevalence of temporomandibular joint disorders in 9-12 year-old boy students in Kerman, southwest Iran. Iran J. Med. Sci., 30(2):91-3, 2005.

Friedman Rubin, P.; Erez, A.; Peretz, B.; Birenboim-Wilensky, R. \& Winocur, E. Prevalence of bruxism and temporomandibular disorders among orphans in southeast Uganda: A gender and age comparison. Cranio, 36(4):243-9, 2018.

Karibe, H.; Shimazu, K.; Okamoto, A.; Kawakami, T.; Kato, Y. \& Warita-Naoi, S. Prevalence and association of self-reported anxiety, pain, and oral parafunctional habits with temporomandibular disorders in Japanese children and adolescents: a cross-sectional survey. B. M. C. Oral Health, 15:8, 2015.

Lavigne, G. J.; Kato, T.; Kolta, A. \& Sessle, B. J. Neurobiological mechanisms involved in sleep bruxism. Crit. Rev. Oral Biol. Med., 14(1):30-46, 2003.

List, T.; Wahlund, K. \& Larsson, B. Psychosocial functioning and dental factors in adolescents with temporomandibular disorders: a case-control study. J. Orofac. Pain, 15(3):218-27, 2001.

McNeill, C. Management of temporomandibular disorders: concepts and controversies. J. Prosthet. Dent., 77(5):510-22, 1997.

Moreno Silva, C. P. Análisis Clínico de la Prevalencia de Trastornos Témporomandibulares en Niños de 8 a 12 años de Edad de la Escuela Diego Abad de Cepeda de la Ciudad de Quito. Tesis de Grado para obtener Título de Odontóloga. Quito, Universidad San Francisco de Quito, 2013.

Riva, R.; Sanguinettí, M.; Rodríguez, A.; Guzzetti, L.; Lorenzo, S.; Álvarez, R. \& Massa F. Prevalencia de trastornos témporo mandibulares y bruxismo en Uruguay. Parte I. Odontoestomatología, 13(17):54-71, 2010.

Rugh, J. D. \& Harlan, J. Nocturnal bruxism and temporomandibular disorders. Adv. Neurol., 49:329-41, 1988.

Sandoval, U. H. \& Fariña, V. M. P. Prevalence of sleep bruxism in children, and its relationship with signs of temporomandibular disorders and daytime oral parafunctions. Int. J. Odontostomat., 10(1):41-7, 2016.
Sena, M. F.;Mesquita, K. S.; Santos, F. R.; Silva, F. W. \& Serrano, K. V. Prevalence of temporomandibular dysfunction in children and adolescents. Rev. Paul. Pediatr., 31(4):538-45, 2013.

Seraj, B.; Ahmadi, R.; Mirkarimi, M.; Ghadimi, S. \& Beheshti, M. Temporomandibular disorders and parafunctional habits in children and adolescence: a review. J. Dent. Tehran Univ. Med. Sci., 6(1):37-45, 2009.

Serrano, A. P.; Fregoso, C. A.; Jiménez, F. J. \& Ocampo, F. Frecuencia de disfunción de la articulación temporomandibular en niños. Rev. Mex. Odontol. Clin., 3(2):4-7, 2009.

Tuerlings, V. \& Limme, M. The prevalence of temporomandibular joint dysfunction in the mixed dentition. Eur. J. Orthod., 26(3):31120, 2004.

Widmalm, S. E.; Christiansen, R. L. \& Gunn, S. M. Oral parafunctions as temporomandibular disorder risk factors in children. Cranio, 13(4):242-6,1995.

Wieckiewicz, M.; Paradowska-Stolarz, A. \& Wieckiewicz, W. Psychosocial aspects of bruxism: the most paramount factor influencing teeth grinding. Biomed. Res. Int., 2014:469187, 2014.

Winocur, E.; Littner, D.; Adams, I. \& Gavish, A. Oral habits and their association with signs and symptoms of temporomandibular disorders in adolescents: a gender comparison. Oral Surg. Oral Med. Oral Pathol. Oral Radiol. Endod.,102(4):482-7, 2006.

Dirección para correspondencia:

Miguel Ángel Rosales Berber

Posgrado de Estomatología Pediátrica

Facultad de Estomatología

Universidad Autónoma de San Luis Potosí, México

Dirección Av. Manuel Nava \#2

Zona Universitaria

San Luis Potosí

MÉXICO

Email: miguel.rosales@uaslp.mx

Recibido : 08-06-2018

Aceptado: 13-08-2018 